\title{
Cholesteric colloidal liquid crystals from phytosterol rod-like particles
}

\author{
Laura Rossi, $\uparrow^{a}$ Stefano Sacanna $^{b}$ and Krassimir P. Velikov*ac \\ Received 15th August 2010, Accepted 5th October 2010 \\ DOI: $10.1039 / \mathrm{c} 0 \mathrm{sm} 00822 \mathrm{~b}$
}

\begin{abstract}
We report the first observation of chiral colloidal liquid crystals of rod-like particles from a low molecular weight organic compoundphytosterols. Based on the particles shape and crystal structure, we attribute this phenomenon to chiral distribution of surface charge on the surface of neighbouring rods.
\end{abstract}

Shape anisotropic (disc-like or rod-like) colloidal particles have been drawing the attention of scientists for many centuries, starting with the use of natural clay particles. ${ }^{1}$ The particular interest in their phase behaviour started already with the work of Onsager who theoretically predicted the occurrence of entropically driven phase transition in suspensions of disc-like particles that leads to the formation of liquid crystal (LC) phases. $^{2}$ Nowadays, available synthetic methods readily provide a vast amount of (organic and inorganic) shape anisotropic colloidal particles, ${ }^{3,4}$ which indeed in many cases give rise to the formation of various LC phases. $^{5-7}$ Interestingly, shape anisotropic colloidal particles can also form a cholesteric (or chiral nematic) LC phase, ${ }^{8-11}$ initially thought to be an exclusive property of molecular LC phases which are typically composed of molecules containing a chiral centre. The latter produces intermolecular forces that favour alignment between molecules at a slight angle to one another in different layers resulting in a long-range chiral order. ${ }^{12}$ Surprisingly, chiral colloidal LC phases have been observed only in the case of shape-anisotropic particles from self-assemblies of large biopolymers (e.g. cellulose ${ }^{13}$ chitin $^{14}$ ) and macromolecules (e.g. viruses ${ }^{15}$ ) and not in low molecular weight organic compounds. Although a lot of research has been done in relation to cholesteric colloidal LCs, the connection between molecular and macroscopic chirality is still not understood..$^{8-11,16}$

Here we report the first observation of a cholesteric LC phase in a colloidal suspension of rod-like particles from phytosterols (PSs): a low molecular weight organic molecule. To our knowledge, this is the first observation of cholesteric LC from colloidal particles made

${ }^{a}$ Unilever $R \& D$ Vlaardingen, Olivier van Noortlaan 120, 3133AT Vlaardingen, The Netherlands. E-mail: Krassimir.Velikov@Unilever. com; Tel: +31104605068

${ }^{b}$ Center for Soft Matter Research, Department of Physics, New York University 4 Washington Place, New York, NY, 10003, USA. E-mail: S.Sacanna@nyu.edu

'Soft Condensed Matter, Debye Institute, Utrecht University, Princeton Plein 5, 3584 CC Utrecht, The Netherlands

$\uparrow$ Current address: van 't Hoff Laboratory for Physical and Colloid Chemistry, Utrecht University, Padualaan 8, $3584 \mathrm{CH}$ Utrecht, The Netherlands. E-mail: L.Rossi@uu.nl of chiral low molecular weight organic molecules. Phytosterols are a group of plant-derived sterols that have structures similar to that of cholesterol (Fig. 1). ${ }^{17}$ Due to their different biochemistry, phytosterols are used as an efficient cholesterol lowering agent in various food products. $^{17,18}$

The procedure developed for the synthesis of phytosterol colloidal particles is based on anti-solvent precipitation. ${ }^{19}$ Transmission electron microscopy (TEM) pictures (Fig. 2) show that the colloidal particles have a rod-like shape with aspect ratios $L / D$ varying between 3 and 20, with $D$ the particle width and length $L$ typically in between $2 \mu \mathrm{m}$ and $3 \mu \mathrm{m} .{ }^{20}$ Phytosterols are known to form rod- and plate-like crystals of hemi- and monohydrates when crystallized from oil solutions $^{21}$ or organic solvents in the presence of water. ${ }^{22}$ Recent studies have shown that cholesterol, which also forms hemi- and monohydrates, forms plate-like particles. ${ }^{23}$ Our rod-like particles were stabilized against aggregation using a non-ionic surfactant polyoxyethylene sorbitan monooleate. A large negative $\zeta$-potential, typically in the order of $-40 \mathrm{mV}$, was found to account for the high particle stability observed in pure water. The negative surface charge is due to the polar hydroxyl groups both from phytosterols and water

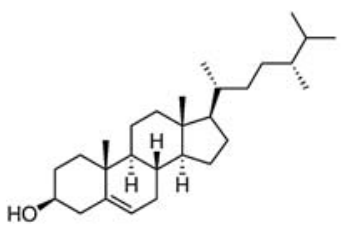

campesterol

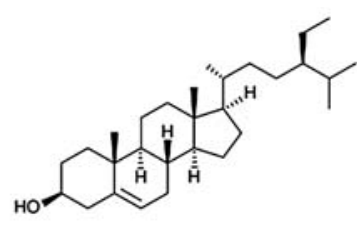

$\beta$-sitosterol
Fig. 1 Chemical structures of some phytosterols.

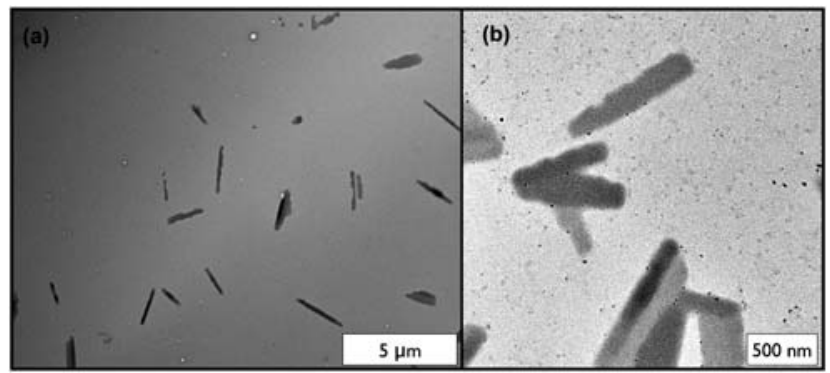

Fig. 2 Typical TEM micrographs showing rod-like phytosterol colloidal particles. Sample (a) was freeze-dried before imaging whereas sample (b) was dried in air. 


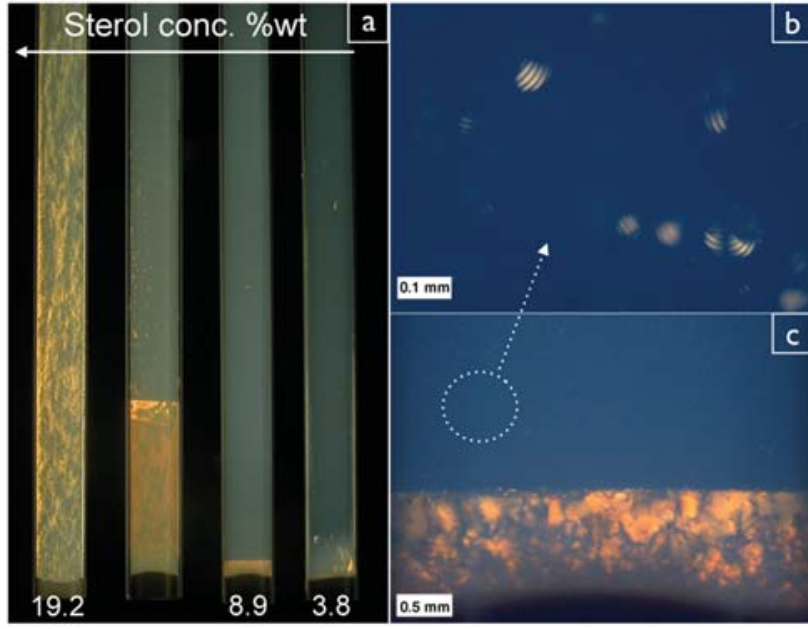

Fig. 3 (a) shows capillaries containing dispersions of colloidal sterol rods at increasing volume fraction. (b) shows a detail of tactoids separating out of the isotropic phase and falling into the birefringent phase coalescing at the interface (c). The pictures are taken between cross polarizers to reveal the birefringent liquid crystal phase.

molecules present at the crystal surface. Similar surface properties were found in the case of cholesterol particles. ${ }^{24,25}$ The surface charge was found to play an important role and despite the presence of the stabilizer, at the isoelectric point $(\mathrm{p} I \approx 2.5$ (ref. 20)) phytosterol particle suspensions aggregate.

Strong flow-birefringence in the phytosterol colloidal dispersion was observed already at relatively low concentration $(\geq 0.7 \% \mathrm{wt})$. The beginning of phase separation was observed at concentration $\geq 3.5 \%$ wt (Fig. 3a). The appearance of an LC phase at this concentration is consistent with the behaviour of other charged rod-like particles of similar aspect ratio such as microcrystalline cellulose ${ }^{26}$ and chitin. ${ }^{27}$ When further concentrated inside flat capillaries, they separated into an isotropic (upper) phase and a highly birefringent (lower) phase (see Fig. 3a). The gravitational settling is negligible in the time scale on which the phase separation occurs (about 12 hours). Depending on the particle concentration, the birefringent phase grows in time fed by tactoids separating from the isotropic upper phase. The volume of the LC phase increases with the increase of particle concentration. The coalescence of tactoids at the interface is clearly visible in Fig. 4. Remarkably, after leaving the capillaries undisturbed for several days, large domains with a characteristic fingerprints patterns grow in the birefringent phase (see Fig. 4).

Identical patterns are sometimes visible also in larger tactoids within the isotropic phase as shown in Fig. $3 b$ and 4 (inset). Extinction and illumination areas (the visible black and white lines) alternate which is caused by the regular change of the direction of nematic layers of rods. These structures are characteristic for the cholesteric, ${ }^{8-11}$ smectic phase, ${ }^{28}$ and buckled nematic liquid crystal phases. ${ }^{29}$ In the smectic liquid crystalline phases the pitch size is on the order of the rod length. ${ }^{30}$ In our experiments the pitch size is constant for all the samples in bulk and in the tactoids and was measured about $15 \mu \mathrm{m}$ in the case of pure water (no added salt), a distance much larger than the average particles length $(2-3 \mu \mathrm{m})$. The large pitch size compared to particle length is consistent with the presence of a cholesteric phase. Buckled nematic liquid crystals with a stripe pattern which resembles the

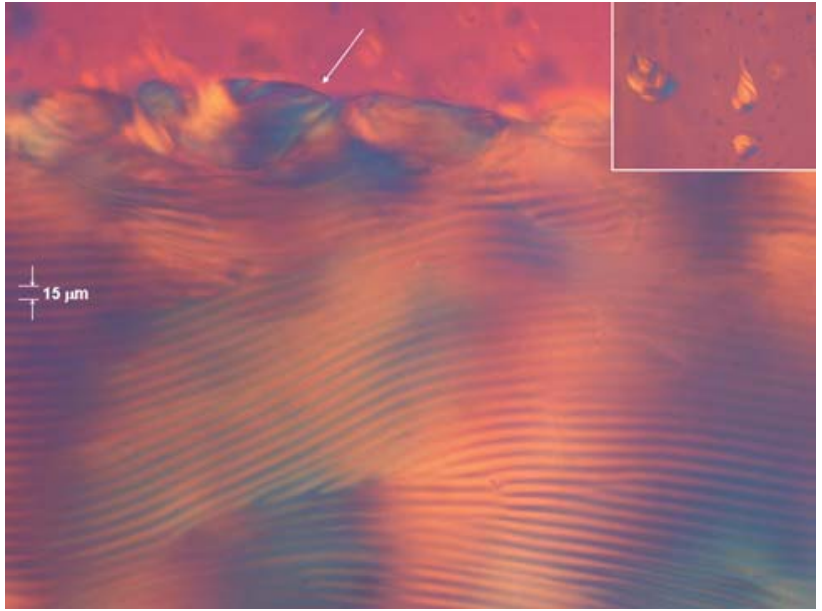

Fig. 4 Optical micrographs (taken between crossed polarizers) showing the characteristic fingerprint patterns of cholesteric LC phase of a concentrated sample of sterol colloidal particles. The arrow highlights a tactoid coalescing at the interface. The inset (top right) shows other tactoids falling down from the isotropic phase.

cholesteric phase are found, for instance, when samples are exposed to an external field ${ }^{31}$ or in nematic gels, ${ }^{29}$ however, both systems are entirely different from the one discussed here. The cholesteric LC phase persists despite the presence of a neutral surfactant layer adsorbed as steric stabilizer on the particles surface pointing to modulated long range electrostatic repulsions. Similar behaviour was observed in PEG-coated rod-like fd virus. ${ }^{11}$

Chirality has been considered as a reason for the formation of molecular LC phases, ${ }^{32,33}$ although recent studies have demonstrated that achiral molecules can also form macroscopically isotropic fluids that possess only short-range orientational and positional order (in which the only macroscopically broken symmetry is chirality). ${ }^{34}$ However, despite of its importance, the link between chirality at the molecular scale and the macroscopic chiral structure in the case of colloidal LC phases is not yet well established. Straley has proposed the first model of the origin of intermolecular twist who considered two screw-like molecules with excluded-volume interactions - "cork-screw". ${ }^{35}$ The formation of cholesteric LCs can be attributed to the formation of screw like (twisted) particles (e.g. uniform chiral microstructures from achiral $\pi$-conjugated organic molecules ${ }^{36}$ ). This model has been used to explain the formation of cholesteric LCs phase in cellulose microfibrils. ${ }^{37}$ The presence of such a chiral twist along the microfibrils was later demonstrated using TEM and atomic force microscopy (AFM) ${ }^{38}$ Screw-like packing is typical for many steroidal molecules and has been found to cause self-assembly of helical ribbons in complex mixtures of cholesterol, non-ionic surfactants, and lipids. ${ }^{39,40}$ In our study, however, we have not found the formation of ribbon-like or other more complex structures, as concluded from TEM observations (Fig. 2). In addition, we did not observe the presence of any additional fine structure in the rod-like particles indicating that this model is not applicable in our case.

In order to explain the formation of cholesteric colloidal liquid crystal from low molecular weight organic molecules, we will consider in more detail the crystal structure of the phytosterol rod-like particles. XRD measurements performed on dried colloidal dispersions indicated that the dry colloidal particles appear to have features of 
hemihydrated crystalline form. ${ }^{20}$ The structure of anhydrous ${ }^{41}$ and monohydrated ${ }^{42}$ sterol crystals was first established for cholesterol. Only very recently the crystal structures were reported for $\beta$-sitosterol hemihydrate ${ }^{22}$ and monohydrate. ${ }^{43}$ Although the crystal structures of both hemihydrate and monohydrate are different, they have a common feature in which sterol molecules form bilayers of thickness 3.4-3.6 nm. The hydroxyl groups and the water molecules are hydrogen bonded to form a sheet at the bilayer interface (see Fig. 5). In all sterol structures the steroid planes appear almost but not parallel, as determined for $\beta$-sitosterol crystals. ${ }^{22}$ Such structure could allow creation of periodic distribution of hydroxyl groups on the surface of the rod like particles. This is equivalent to the creation of patchy rod-like particles with chiral distribution of alternating polar and apolar stripes, which can promote chiral distribution of surface charge. Phytosterol particles are negatively charged ${ }^{20}$ due to partial deprotonation of $\mathrm{OH}$ groups and water molecules on the particle surface, as observed in the case of cholesterol. ${ }^{44}$ Such surface charge heterogeneities or domains may arise from the competition of electrostatic forces with segregating forces such as steric, van der Waals, and hydrogen bonding interactions. ${ }^{16,45}$

Based on the above considerations, here we hypothesize that the crystal symmetry of both hemi- and monohydrate sterol crystals could induce helicity in the distribution of polar groups and ions on the surface of the rod-like particles. The phytosterol rod-like particles therefore appear chiral objects with a helical charge distribution on the particles surface (i.e. "molecularly patchy particles") and subsequent formation of a cholesteric phase can be attributed to its chirality. A similar model was proposed by Orts et al. for cellulose LCs where inhomogeneous distribution of surface charge can cause the formation of chiral colloidal LCs. ${ }^{37,46-48}$ The same mechanism has been discussed in relation to fd virus ${ }^{11}$ and chitin ${ }^{46}$ particles. The most recent and advanced theories of cholesteric assemblies of DNA modelled as charged rods also predict cholesteric phase existence which coincides with the domain of strong biaxial correlations between nearest-neighbour molecules. ${ }^{49}$ The theory interprets the cholesteric organization as the result of the competition of steric and electrostatic repulsion between charged helices. It also suggests that the macroscopic properties of the cholesteric phase are affected by the pattern of ion adsorption. The same mechanism, supported by coarse-grained modelling, was established for cholesteric phase from charged filamentous virus M13. ${ }^{8}$ The formation of a cholesteric LC is explained as the result of the competition between contributions of opposite handedness, deriving from best packing of viral particles and electrostatic interparticle repulsions.
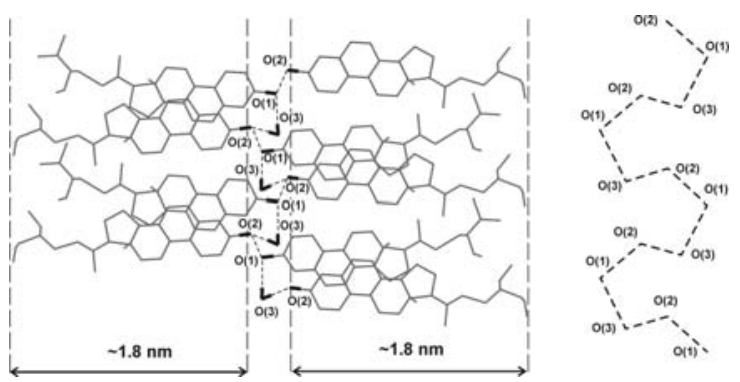

Fig. 5 A diagrammatic view of the $\beta$-sitosterol hemihydrate crystal structure indicating the hydrogen bonding (left) and their bonding and distance (right). ${ }^{22}$ The recurring areas rich in OH-groups and water molecules are separated by a distance of $\sim 3.6 \mathrm{~nm}$.
In conclusion, we report the first observation of chiral colloidal crystals of rod-like particles from a low molecular weight organic compound - phytosterols. Based on the particles shape and crystal structure, we attribute this phenomenon to chiral distribution of the phytosterol and water molecules on the surface of neighbouring rods. We assume that helicity is preserved during the precipitation and the particles have chiral distribution of surface charge, which contributes to the formation of cholesteric colloidal LC phases. Such LCs can be used as an environmentally friendly and convenient model system to study phase behaviour of shape-anisotropic particles. Colloidal LCs from phytosterol colloidal particles can find application in displays as biodegradable environmentally friendly materials which have lower density in comparison to rod-like particles from inorganic materials ${ }^{6}$ and have better (chemical and microbial) stability in comparison to protein/polypeptide based rodlike particles. Importantly, sterols allow several chemical modifications, which can be used to design particles with tunable chiral molecular patches, which can help to establish in detail the mechanism of formation of cholesteric LC phases and reveal the role of molecular chirality.

\section{Experimental}

\section{Materials}

Phytosterols (mixture with $c a$. 10\% campesterol, $c a .75 \% \beta$-sitosterol) were obtained from Acros Organics. Polyoxyethylene sorbitan monooleate (Tween®80) was obtained from MERK. Ethanol used for synthesis (Royal Nedalco) was of analytical reagent quality. Deionized water purified by a Millipore purification system (MILLIPORE Synergy 185) was used in all reactions. All chemicals were used as received.

\section{Sample preparation}

Colloidal particles were synthesized as describe in ref. 20. Phytosterol was dissolved in ethanol and particle precipitation was induced by rapid mixing of this solution with water. The organic phase used in most experiments consisted of $40 \mathrm{~g}$ of ethanol with a variable amount of sterol and stabilizers. The ethanolic solution was quickly injected, with a $50 \mathrm{~mL}$ syringe without needle, in the aqueous phase consisting in $360 \mathrm{~g}$ of water or water/ethanol mixture under vigorous stirring (using Silverson L4RT-A stirrer usually working at 3000-4000 rpm). After particles precipitation the dispersion was kept under stirring for additional 2-3 minutes. The dispersions were then purified and concentrated by solvent evaporation using a rotavapor (BUCHI, R-114).

\section{Optical microscopy}

The concentrated dispersions were studied in flat optical capillaries (VitroCom) with internal dimensions of $200 \mu \mathrm{m} \times 4 \mathrm{~mm} \times 8 \mathrm{~cm}$ sealed with an epoxy glue to prevent solvent evaporation. The samples were left undisturbed on a marble table in a dark thermostatted room and were investigated with a Nikon LV100 polarizing microscope, equipped with a QImaging MicroPublisher 5 megapixel CCD camera. 


\section{Electron microscopy}

The particle size and morphology were investigated by transmission electron microscopy (TEM) using a Philips TECNAI 20 operating at $200 \mathrm{kV}$. TEM samples were prepared by drying a drop of diluted dispersion on carbon-coated copper grids. In order to minimize drying effects, few TEM samples were first rapidly gassified by a fast temperature quench in liquid nitrogen and then freeze-dried under vacuum.

\section{Acknowledgements}

The authors would like to thank J. Hazekamp for his assistance with TEM and A. A. Verhoeff for her assistance with optical microscopy and stimulating discussions with A. Philipse and M. Bonn. This work is financially supported by Food Nutrition Delta program grant DFN0642300.

\section{References}

1 H. Zocher, Z. Anorg. Allg. Chem., 1925, 147, 91.

2 L. Onsager, Ann. N. Y. Acad. Sci., 1949, 51, 627.

3 C. N. R. Rao, F. L. Deepak, G. Gundiah and A. Govindaraj, Prog. Solid State Chem., 2003, 31, 5.

4 S. C. Glotzer and M. J. Solomon, Nat. Mater., 2007, 6, 557.

5 S. J. Zhang and S. Kumar, Small, 2008, 4, 1270.

6 P. Davidson and J. C. P. Gabriel, Curr. Opin. Colloid Interface Sci., 2005, 9, 377.

7 J. C. P. Gabriel and P. Davidson, Adv. Mater., 2000, 12, 9.

8 F. Tombolato, A. Ferrarini and E. Grelet, Phys. Rev. Lett., 2006, 96, 258302-1-258302-4

9 Z. Dogic and S. Fraden, Curr. Opin. Colloid Interface Sci., 2006, 11, 47.

10 E. Barry, Z. Hensel, Z. Dogic, M. Shribak and R. Oldenbourg, Phys. Rev. Lett., 2006, 96, 018305.

11 E. Grelet and S. Fraden, Phys. Rev. Lett., 2003, 90, 198302.

12 H. S. Kitzerow, C. Bahr and S. Chandrasekhar, Chirality in Liquid Crystals, Springer, 2001.

13 M. M. D. Lima and R. Borsali, Macromol. Rapid Commun., 2004, 25, 771.

14 E. Belamie and M. M. Giraud-Guille, Trends in Colloid and Interface Science XVII, 2004, vol. 126, p. 159.

15 Z. Dogic and S. Fraden, Langmuir, 2000, 16, 7820.

16 K. L. Kohlstedt, F. J. Solis, G. Vernizzi and M. O. de la Cruz, Phys. Rev. Lett., 2007, 99, 030602.

17 R. E. Ostlund, Curr. Opin. Lipidol., 2004, 15, 37.

18 A. Ambring, P. Friberg, M. Axelsen, M. Laffrezen, M. R. Taskinen, S. Basu and M. Johansson, Clin. Sci., 2004, 106, 519.

19 H. Fessi, F. Puisieux, J. P. Devissaguet, N. Ammoury and S. Benita, Int. J. Pharm., 1989, 55, R1-R4.
20 L. Rossi, J. W. M. S. ten Hoorn, S. M. Melnikov and K. P. Velikov, Soft Matter, 2010, 6, 928.

21 L. I. Christiansen, J. T. Rantanen, A. K. von Bonsdorff, M. A. Karjalainen and J. K. Yliruusi, Eur. J. Pharm. Sci., 2002, 15, 261.

22 H. Kawachi, R. Tanaka, M. Hirano, K. Igarashi and H. Ooshima, J. Chem. Eng. Jpn., 2006, 39, 869.

23 V. Uskokovic, Steroids, 2008, 73, 356.

24 L. Mukhopadhyay, P. K. Bhattacharyya, A. R. Das and S. P. Moulik, Colloid Polym. Sci., 1993, 271, 793.

25 R. Shabd, B. M. Upadhyay and S. B. Singh, Carbohydr. Res., 1981, 98, 123.

26 J. Araki and S. Kuga, Langmuir, 2001, 17, 4493.

27 E. Belamie, P. Davidson and M. M. Giraud-Guille, J. Phys. Chem. B, 2004, 108, 14991

28 S. A. Langer and J. P. Sethna, Phys. Rev. A, 1986, 34, 5035.

29 R. Verduzco, G. N. Meng, J. A. Kornfield and R. B. Meyer, Phys. Rev. Lett., 2006, 96, 147802.

30 H. Maeda and Y. Maeda, J. Chem. Phys., 2004, 121, 12655.

31 M. R. Kuzma, Phys. Rev. Lett., 1986, 57, 349.

32 E. Grelet, B. Pansu, M. H. Li and H. T. Nguyen, Phys. Rev. E: Stat., Nonlinear, Soft Matter Phys., 2002, 65, 050701.

33 P. G. de Gennes and J. Prost, The Physics of Liquid Crystals, Clarendon, Oxford, 1993.

34 L. E. Hough, M. Spannuth, M. Nakata, D. A. Coleman, C. D. Jones, G. Dantlgraber, C. Tschierske, J. Watanabe, E. Korblova, D. M. Walba, J. E. Maclennan, M. A. Glaser and N. A. Clark, Science, 2009, 325, 452.

35 J. P. Straley, Phys. Rev. A: At., Mol., Opt. Phys., 1976, 14, 1835.

36 H. B. Chen, Y. Zhou, J. Yin, J. Yan, Y. G. Ma, L. Wang, Y. Cao, J. Wang and J. Pei, Langmuir, 2009, 25, 5459.

37 W. J. Orts, L. Godbout, R. H. Marchessault and J. F. Revol, Macromolecules, 1998, 31, 5717.

38 S. J. Hanley, J. F. Revol, L. Godbout and D. G. Gray, Cellulose, 1997, 4, 209.

39 F. M. Konikoff, D. S. Chung, J. M. Donovan, D. M. Small and M. C. Carey, J. Clin. Invest., 1992, 90, 1155.

40 Y. V. Zastavker, N. Asherie, A. Lomakin, J. Pande, J. M. Donovan, J. M. Schnur and G. B. Benedek, Proc. Natl. Acad. Sci. U. S. A., 1999, 96, 7883.

41 H. S. Shieh, L. G. Hoard and C. E. Nordman, Nature, 1977, 267, 287.

42 B. M. Craven, Nature, 1976, 260, 727.

43 G. Argay, A. Kalman, S. Vladimirov, D. Zivanovstakic and B. Ribar, Z. Kristallogr., 1996, 211, 725.

44 V. Uskokovic, Mater. Manuf. Processes, 2008, 23, 620.

45 G. Vernizzi, K. L. Kohlstedt and M. O. de la Cruz, Soft Matter, 2009, $5,736$.

46 J. F. Revol, H. Bradford, J. Giasson, R. H. Marchessault and D. G. Gray, Int. J. Biol. Macromol., 1992, 14, 170.

47 L. Heux, G. Chauve and C. Bonini, Langmuir, 2000, 16, 8210.

48 J. Araki, M. Wada and S. Kuga, Langmuir, 2001, 17, 21.

49 A. A. Kornyshev, S. Leikin and S. V. Malinin, Eur. Phys. J. E: Soft Matter Biol. Phys., 2002, 7, 83. 